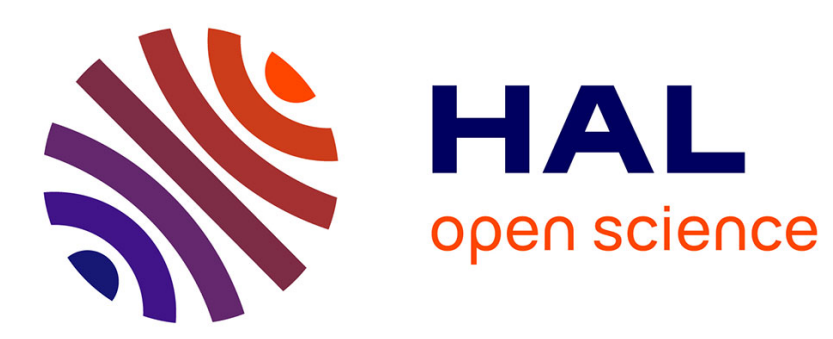

\title{
A Threshold-Based Approach for Joint Active User Selection and Feedback in MISO Downlink Systems
}

Apostolos Destounis, Mohamad Assaad, Mérouane Debbah, Bessem Sayadi

\section{To cite this version:}

Apostolos Destounis, Mohamad Assaad, Mérouane Debbah, Bessem Sayadi. A ThresholdBased Approach for Joint Active User Selection and Feedback in MISO Downlink Systems. IEEE International Conference on Communications (ICC), Jun 2015, London, United Kingdom. 10.1109/icc.2015.7248762 . hal-01242581

\section{HAL Id: hal-01242581 \\ https://hal.science/hal-01242581}

Submitted on 13 Dec 2015

HAL is a multi-disciplinary open access archive for the deposit and dissemination of scientific research documents, whether they are published or not. The documents may come from teaching and research institutions in France or abroad, or from public or private research centers.
L'archive ouverte pluridisciplinaire $\mathbf{H A L}$, est destinée au dépôt et à la diffusion de documents scientifiques de niveau recherche, publiés ou non, émanant des établissements d'enseignement et de recherche français ou étrangers, des laboratoires publics ou privés. 


\title{
A Threshold-Based Approach for Joint Active User Selection and Feedback in MISO Downlink Systems
}

\author{
Apostolos Destounis*, Mohamad Assaad*, Mérouane Debbah*, Bessem Sayadi ${ }^{\ddagger}$ \\ ${ }^{*}$ Centrale-Supélec, 3 rue Joliot-Curie, 91192, Gif-sur-Yvette, cedex. France \\ email: \{apostolos.destounis, mohamad.assaad, merouane.debbah\}@ centralesupelec.fr \\ $\ddagger$ Alcatel-Lucent Bell Labs France, Route de Villejust, 91620, Nozay, cedex. France \\ email: bessem.sayadi@alcatel-lucent.com
}

\begin{abstract}
In this paper we study the downlink of a TDD (Time Division Duplex) single cell system where the Base Station (BS) employs multiple antennas to serve the users taking into account the traffic patterns. The BS chooses each slot the users to be active, and serves them using Zero Forcing (ZF) precoding. This requires the knowledge of the users' channels which is assumed to be performed e.g. via uplink training. Due to the channel acquisition overhead, only a subset of users must be active at each timeslot (depending on traffic patterns and channel states). In this paper, we develop an active user selection strategy where the base station sets a given threshold for the channel gain of the users. Then, only the users that have their channel gain higher than the threshold send their training sequence and are then considered to be served. The base station estimates the channel states of these users and, due to channel reciprocity, uses these channel states to transmit data via $\mathrm{ZF}$ precoding. With appropriate signaling and threshold selection, which adapt to the queuing behavior of the users, we prove that our proposed method achieves a larger stability region than the baseline centralized policy where the BS selects the users based on channel statistics and queue lengths. The performance of the threshold-based method is illustrated via simulations, where we can observe a tradeoff between the expansion of the stability region and delay performance.
\end{abstract}

\section{INTRODUCTION}

The use of multiple antennas at the transmitters is recognized as one of the key enabling technologies in order to improve the performance of wireless systems [1]. This is mainly due to the ability of a transmitter to serve multiple users simultaneously in the same time-frequency block. Initial works on MIMO systems have been focused on aspects like spectral efficiency, however it is of great theoretical and practical interest to see how the introduction of multiple antennas affects the queueing behaviour of the users [2]. An algorithm based on iterative waterfilling to solve the scheduling and power control problem in order to achieve maximum stability region has been proposed in [3]. However, these works employ dirty paper coding and successive encoding/decoding, that are complex to implement in practice. Linear precoding techniques, with lower complexity are more widely used. In particular, a popular technique is Zero Forcing (ZF), where interference among users is suppressed. This is a relatively simple technique, however it performs well in many cases of interest and captures the fundamental aspects of MIMO communications. On the other hand, MIMO systems achieve tremendous gain under the assumption that the channel states are known to the BS. This can be done only by feedback or training from the users. Among these techniques, training in TDD mode is considered the most prominent since the length of the training sequences does not depend on the number of BS antennas. However, in this case the time of the slot devoted to training is proportional to the number of users that perform the uplink training. Therefore, serving many users at the same timeslot requires a greater fraction of the timeslot to be spent for training, leaving a smaller part of the slot available for data transmission.

Limited feedback in MIMO systems has been a widely studied topic, see e.g. [4], [5] and references therein, however most works address problems like maximizing the sum rate of the system and they do not take into account the traffic demands of the users. The work related more to our present paper is the one in [6], where the authors deal with the effect of imperfect feedback (due to quantization) on the stability region of a system with $\mathrm{ZF}$ precoding. The authors do not take into account the resources needed for feedback and they employ a centralized scheme where the BS selects the users to serve based only on the queue lengths. This has the drawback of possibly selecting users with bad channels to serve.

In this paper we study the problem of maximizing the stability region of the users' queues in the downlink of a multiuser MISO wireless system, where Channel State Information acquisition is done via uplink training and the BS serves users via Zero Forcing precoding. We consider CSI estimation without errors in order to focus on the impact of the training overhead on the performance of the system. We propose a scheme where the BS sets periodically a threshold and a preferred number of users to perform training. The users having their channel gain higher than the threshold will send their training sequence which ensures that each user who gets served (after ZF precoding) has a channel realization with gain above the threshold.

At this point, we would like to mention that the idea of threshold based feedback has been used in SISO systems in order to reduce the feedback overhead while selecting a "good" user to schedule. It was initiated in [7], [8] for sumrate maximization without considering the queues of the users. The recent work [9] addressed the problem of stability region expansion. Therein, the channel state of the user with the 
largest queue is used as threshold. The above frameworks cannot be simply extended to MIMO systems. In fact, in MIMO systems using ZF precoding (or in more general beamforming), the bit rate of each user does not depend only on its channel conditions but also on the channel realization of the other users that get served simultaneously, making the problem more challenging. Finally, authors in [10] employ similar ideas for a multiuser MIMO system where the receivers employ Zero Forcing. Apart from focusing on the receivers and not the precoder at the transmitter, the work in [10] addresses sum rate maximization, so the traffic processes of the users are not taken into account. More in this direction, a recent work [11] showed that feedback policies exploiting the fact that the users know their channel can enlarge the stability region for a two-user system, under the idealized assumption that contention without collisions ispossible.

The rest of the paper is organized as follows: We present the system model in Section II. Section III describes the operation and stability region of a centralized policy, which selects users based solely on queue lengths and channel statistics while Section IV presents the threshold-based policy. Finally, Section $\mathrm{V}$ provides simulation results and Section VI concludes the paper.

Notations: Throughout the paper, uppercase boldface symbols represent matrices while lowercase boldface symbols represent column vectors. $\mathbf{x}$ represents a vector with elements $x_{k}$ and $\|\mathbf{x}\|$ is it Euclidean norm. In addition, $\mathbf{I}_{N}$ denotes the identity matrix of size $N$. Given a matrix $\mathbf{A}, \mathbf{A}^{H}$ denotes its Hermitian and $\mathbf{A}^{-1}$ its inverse. The notation $\mathcal{C N}\{\mu, \mathbf{R}\}$ is used for a vector-valued complex normal circularly symmetric random variable with mean $\mu$ and covariance matrix $\mathbf{R}$, while $\mathcal{C H}$ denotes the convex hull operation. We use $\Gamma(N)$ to denote the Gamma function with parameter $N, B(a, b)$ to denote the Beta function with parameters $a$ and $b$ and $I_{B}(x ; a, b)$ to denote the upper incomplete Beta function with parameters $a, b$ at point $x$. Finally, $f_{N}(x)=\frac{x^{N-1} e^{-x / 2}}{\Gamma(N)}$ and $\mathbb{1}_{\{A\}}$ denotes the indicator function of the event $A$.

\section{SySTEM MODEL}

\section{A. Physical layer model}

We consider a single cell downlink system where the Base Station (BS) is equipped with $N$ antennas and serves $K$ singleantenna users. Time is slotted and each timeslot has a duration of $T_{s}$ channel uses. Channels are assumed to follow the i.i.d. Rayleigh block fading model, that is $\mathbf{h}_{k}(t) \sim \mathcal{C N}\left(\mathbf{0}, \mathbf{I}_{N}\right)$ for any user $k$ and timeslot $t^{1}$. This implies that the double of the channel magnitudes $2 g_{k}(t)=2\left\|\mathbf{h}_{k}(t)\right\|^{2}$ follow a chisquared distribution with $2 N$ degrees of freedom while the (unitary) vectors $\mathbf{u}_{k}(t)=\frac{\mathbf{h}_{k}(t)}{\left\|\mathbf{h}_{k}(t)\right\|}$ are isotropically distributed. For the rest of the paper we will denote $p(x)=\mathbb{P}\left\{g_{k}(t)>x\right\}$. Without loss of generality, we assume for the noise $n_{k}(t) \sim$ $\mathcal{C N}(0,1)$. As for any linear precoder, the signal received at

\footnotetext{
${ }^{1}$ In practice this can be achieved by using power control to compensate for the large scale fading.
}

user $k$ is

$$
y_{k}(t)=\mathbf{h}_{k}^{H}(t) \mathbf{w}_{k}(t) s_{k}(t)+\sum_{j \neq k} \mathbf{h}_{k}^{H}(t) \mathbf{w}_{j}(t) s_{j}(t)+n_{k}(t),
$$

where $s_{j}(t)$ is the information symbols sent to user $j, \mathbf{w}_{k}(t)$ is the precoding applied to user $k(\mathrm{ZF})$ and $\sum_{k=1}^{K}\left\|\mathbf{w}_{k}(t)\right\|^{2}=$ $P$.

At each slot $t$, the BS selects the maximum number of users that can perform uplink training (for the BS to estimate the channels). In Sections III and IV we present in detail schemes to select the users the BS will serve in the timeslot (the "active" users in the MIMO literature). For a set $\mathcal{F}$ of active users, $\beta F$ channel uses are needed for the lengths of the training sequences $(F=\operatorname{card}(\mathcal{F}), \beta \geq 1)$. The BS serves the active users using $\mathrm{ZF}$ precoding and allocating equal power to each, i.e. $\left\|\mathbf{w}_{k}(t)\right\|^{2}=P / F, \forall k \in \mathcal{F}$. Finally, a scheduled user gets a rate $r_{k}(t)$ of $R$ bits per channel use if the corresponding SNR exceeds a threshold $S$ for correct decoding. Before continuing the description of the system model, we provide the following result from [12] that will be of great help in the analysis done later on in the paper:

Lemma 1. The probability that the received SNR at user $k$ exceeds $S$ given its channel magnitude and that this user and other $F-1$ users are scheduled (with $F \geq 2$ ) is given by

$$
\begin{aligned}
& \mathbb{P}\left\{S N R_{k}(t)>S \mid g_{k}(t), F\right\}= \\
& 1-I_{B}\left(\frac{F}{P g_{k}(t)} S ; N-F+1, F-1\right),
\end{aligned}
$$

if $S \leq \frac{g_{k}(t) P}{F}$, and zero otherwise.

We now proceed to discuss how the physical layer affects the queueing performance of the system.

\section{B. Queueing model}

We assume that each user has an incoming arrival process $a_{k}(t)$, i.i.d. in time with mean $\lambda_{k}$ bits per slot and there exists $A_{\max }<\infty$ such that $a_{k}(t)<A_{\max }$ almost surely. Traffic arriving for user $k$ gets buffered in a respective queue in the BS until it gets transmitted; denote $q_{k}(t)$ the length of this queue at the beginning of slot $t$. Assuming $\tau(t)$ channel uses needed for control information (e.g. uplink training) at slot $t$, if user $k$ is scheduled $\mu_{k}(t)=\left(T_{s}-\tau(t)\right) r_{k}(t)$ bits are removed from its buffer (where $T_{s}$ is the time slot length), meaning that its queue evolves as

$$
q_{k}(t+1)=\left[q_{k}(t)-\mu_{k}(t)\right]^{+}+a_{k}(t), t \geq 0 .
$$

Due to the use of multiple antennas, up to $F$ users can be served in parallel at the same time-frequency block. If many users send their training sequences, the BS will acquire the channel states of a high number of users and will then select the best $F$ users among them which will increase the total bit rate. On the other hand, a bigger part of the timeslot is allocated to uplink training, which will reduce the total bit rate. Clearly, a subset of users must perform uplink training which results in the following challenging problem: how to 
select the users that must perform uplink training and what is the maximum number of these users at each slot $t$. In order to provide an answer to the aforementioned problem, we consider a metric that takes into account the traffic pattern. In fact, we are interested in the stability of the queues, which is defined as follows,

Definition 2. The system is called (strongly) stable if $\lim _{T \rightarrow \infty} \sup \frac{1}{T} \sum_{t=0}^{T-1} q_{k}(t)<\infty, \forall k \in \mathcal{K}$

Intuitively, stability means that the average delay seen by every user is finite, it is thus a relevant metric for data applications. The concept of a stability region is as follows:

Definition 3. Stability region of the system is the set of mean arrival rate vectors $\lambda$ for which the system is stable.

In this work we are interested in finding a strategy that selects the active users who will perform uplink training in such a way to maximize the stability region of the system. From a practical point of view, this implies maximizing the set of arrival rates that the system can sustain for users with delaytolerant applications.

Finally, we provide the following result will also be useful in this paper (see [12] for a proof):

Lemma 4. Define a system $\hat{\mathbf{q}}(m), m=0,1,2, \ldots$ as the original queueing system sampled every $T$ slots, i.e. $\hat{\mathbf{q}}(m)=$ $\mathbf{q}(m T)$. Then the system $\hat{\mathbf{q}}(m)$ is stable if, and only if, the system $\mathbf{q}(t)$ is stable.

\section{Baseline: Centralized Policy}

The baseline approach, followed in existing 3GPP LTE systems and in recent works like [6] and [13], is that the BS is the one selecting the users from which to receive feedback. This means that the BS selects the users to feed back (or train in our context) based only on the queue lengths and channel statistics, as done e.g. in [6]. More precisely, in the beginning of the slot the BS sends a pilot signal of duration $\beta_{p}$ channel uses and then the Identification numbers (IDs) of the users that will perform training. For $F$ users to be scheduled (for feedback) and a control rate of $R_{0}$ bits/channel use, this takes $\beta_{c}=\frac{F \log _{2} K}{R_{0}}$ channel uses. Denoting $\bar{r}(F)$ the average rate in bits/channel uses a scheduled user gets if $F$ users in total are scheduled, we can show using Lemma 1 that, in the case of Rayleigh fading

$\bar{r}(F)=\left\{\begin{array}{l}R \int_{S / P}^{+\infty} f_{N}(z) d z, F=1 \\ R \int_{S / P}^{+\infty} f_{N}(z)\left(1-I_{B}\left(\frac{F S}{P z} ; N-F+1, F-1\right)\right) d z \\ , F>1\end{array}\right.$

Therefore, the mean service rate a user will get if scheduled along with $F-1$ others is

$$
\bar{\mu}(F)=\left[T_{s}-\left(\beta_{p}+\left(\beta+\frac{\log _{2} K}{R_{0}}\right) F\right)\right]^{+} \bar{r}(F) .
$$

The centralized policy then consists in scheduling (for both feedback and ZF precoding) at timeslot $t$ the $F_{c}^{*}(t)$ largest queues where:

$$
F_{c}^{*}(t)=\arg \max _{1 \leq F \leq F_{\max }}\left\{\bar{\mu}(F) \sum_{i=1}^{F} q_{k(i)}(t)\right\} .
$$

In other words, this policy selects the best $F_{c}^{*}(t)$ users that must perform uplink training based on the queues and channel statistics. Once these users send their uplink training sequences, the BS estimates the channel of these users and applies a ZF precoding. This policy is inspired by MaxWeight [14] and is actually the one that maximizes the expectation of the quantity $\sum_{k=1}^{K} q_{k}(t) \mu_{k}(t)$ over the channel statistics. Based on this observation, we can show the following:

Theorem 5. The stability region of the centralized policy is $\Lambda_{c}=\mathcal{C H}\left\{\Lambda_{c}^{\prime}(1), \ldots, \Lambda_{c}^{\prime}\left(F_{\max }\right)\right\}$, where $\Lambda_{c}^{\prime}(F)$ is the convex hull of all points in $\mathbb{R}^{K}$ with $F$ elements equal to $\bar{\mu}(F)$ and the others equal to zero.

The above centralized policy is simple to implement, however it selects the users based on the channel statistics and not the real channel state realizations. A user with bad instantaneous channel can then perform uplink training which results in wasting the resources of the system. For example, if $F_{c}^{*}(t)=2$ and the channel of one user $k(1)$ is such that $\frac{P g_{k(1)}(t)}{2}<S$, then the service rate of this user in the slot is zero. In this case, a better solution may have been to select another user instead of $k(1)$, or schedule user $k(2)$ only.

\section{Threshold-BASEd POLICY}

The shortcomings of the centralized policy come from the fact that the channel realizations do not enter the scheduling decision, therefore users with very bad channels may perform training and may be scheduled. On the other hand, note that the users know their channel state realization through the downlink pilot. The main idea, therefore, of the proposed scheme is that the BS sets a (preferred) number of users to be served, $F$, among a set of users $\overline{\mathcal{K}} \subseteq \mathcal{K}$ and a threshold $G$ for the channel gain; only users with $g_{k}(t)>G$ can train and can get therefore served. The control parameters of the algorithm are therefore: the set of candidate users to be served, $\overline{\mathcal{K}}$, the preferred number of users in this set to be served, $F$, and the threshold $G$. These parameters should be dynamic in order to adapt to the evolution of the queue lengths. The thresholds belong to some set $\mathcal{G}$.

In more detail, the main idea of the threshold-based policy is that the BS uses slots $0, T, 2 T, . ., m T, \ldots$ for signaling and setting the control parameters to be used for the next $T-1$ slots. $T$ is a (positive and possibly high) constant whose role in the performance of the algorithm will be examined in the next subsection. There are therefore two distinct phases:

1) Broadcast phase $(\mathbf{t}=\mathbf{0}, \mathbf{T}, \mathbf{2 T}, \ldots)$ : At slot $m T$ the BS broadcasts IDs of the $\bar{K}(m)$ users with the biggest queue lengths (the set $\overline{\mathcal{K}}(m)$ ) ordered by decreasing queue length, the preferred number of users to be served $F(m)$ and the threshold $G(m)$, given as the solution of (2) that follows.

2) Uplink Training phase in each time slot: In all $T-1$ slots 
(i.e. between slots $m T$ and $(m+1) T)$, the user selection for uplink training is done as follows. A portion of the time slot is reserved to receive information from the users in $\overline{\mathcal{K}}(m)$ about their channels. This portion of time is divided into minislots of total number equal to the number of users in $\bar{K}(m)$. In each minislot (which lasts one channel use), a user sends a signal (e.g. 1 bit signal) if its channel gain is above $G(m)$ and does nothing otherwise. The minislots are shared among the users in $\overline{\mathcal{K}}(m)$ in a TDMA manner according to the ordering broadcasted by the $\mathrm{BS}$ at slot $m T$. The procedure stops in the following cases:

i. When $F(m)$ users with channel above the threshold are found. In this case these users get scheduled for uplink training and the user selection period lasts $L(G(m), F(m))$ channel uses. These $F(m)$ will send then their uplink training sequences that will last $\beta F(m)$ channel uses. The remaining time in the current time slot is then used for data transmission (we will see that even with this additional signaling the performance of this algorithm is better than the baseline user selection algorithm).

ii. When all users in $\overline{\mathcal{K}}$ have sent the 1 bit signal (as above) and $U \leq F(m)$ users have signaled that their channel gain is above the threshold. In this case, the user selection period will have taken up $\bar{K}$ channel uses. These $U$ users will be active in the timeslot and will then send their training sequences (i.e. $\beta U$ channel uses are used for uplink training). The remainder of the time slot is then used for data transmission.

\section{A. Selection of Parameters $(F(m), \bar{K}(m), G(m))$}

In order to obtain the way the aforementioned parameters are selected, let us first define as $\bar{r}_{d}(U, G)$ the average rate in bits per channel use that a user in the ranking will get if scheduled according to the threshold based scheme along with $U-1>0$ other users and threshold $G$. Using Lemma 1 and the fact that we have Rayleigh fading there is:

$$
\begin{aligned}
\bar{r}_{d}(U, G)= & R \frac{1}{p(G)} \int_{G}^{+\infty} f_{N}(z)(1- \\
& \left.I_{B}\left(\frac{U S}{P z} ; N-U+1, U-1\right)\right) d z,
\end{aligned}
$$

and, for $U=1$ :

$$
\bar{r}_{d}(1, G)=\left\{\begin{array}{l}
R, P G>S \\
R\left(1-\frac{\mathbb{P}\left\{G \leq g_{k}(t) \leq S / P\right\}}{p(G)}\right), P G \leq S
\end{array}\right.
$$

From the discussion above and denoting in addition

$$
M(i, m, U)=\sum_{j=\max \{0, U-1-m+i\}}^{\min \{U-2, i-1\}}\left(\begin{array}{c}
i-1 \\
j
\end{array}\right)\left(\begin{array}{c}
m-i-1 \\
U-(j-2)
\end{array}\right)
$$

we can show the following (the proof is in the Appendix):

Lemma 6. Let the threshold for the channel gain be $G$ and that $F$ users out of the first $\bar{K}$ users are to be scheduled. If $i \leq \bar{K}$, the mean service rate given to the $i-$ th user in the ranking is given as

$$
\begin{aligned}
& \bar{\mu}_{i}(1, \bar{K}, G)= \\
& {\left[T_{s}-\left(\beta_{p}+i+\beta\right)\right]^{+}(1-p(G))^{i-1} p(G) \bar{r}_{d}(1, G), \text { if } F=1}
\end{aligned}
$$

and, if $F>1$

$$
\begin{aligned}
& \bar{\mu}_{i}(F, \bar{K}, G)= \\
& \bar{r}_{d}(F, G)\left[T_{s}-\beta_{p}-i-\beta F\right]^{+} p^{F}(G)(1-p(G))^{i-F} \mathbb{1}_{\{F \leq i\}} \\
& +\bar{r}_{d}(F, G) p^{F}(G) \sum_{m=\max [F, i+1]}^{\bar{K}}\left[T_{s}-\beta_{p}-m-\beta F\right]^{+} \\
& M(i, m, U)(1-p(G))^{m-F}+\sum_{F^{\prime}=1}^{F-1}\left(\begin{array}{l}
\bar{K}-1 \\
F^{\prime}-1
\end{array}\right)\left[T_{s}-\beta_{p}-\right. \\
& \left.\bar{K}-\beta F^{\prime}\right]^{+} p^{F^{\prime}}\left(g^{*}\right)\left(1-p\left(g^{*}\right)\right)^{\bar{K}-F^{\prime}} \bar{r}_{d}\left(F^{\prime}, G\right) .
\end{aligned}
$$

In addition, $\mu_{i}(F, \bar{K}, G)=0$ for $i>\bar{K}$.

Denote now $\mathcal{G}$ the set of thresholds. The threshold - based algorithm consists in the BS selecting at timeslot $m T$ a number of users $F(m)$ and a threshold $G(m)$ to be used in the next $T-1$ slots such that

$$
\begin{aligned}
(F(m), \bar{K}(m), G(m))= & \arg \max _{F \in\left\{1, . ., F_{\max }\right\}, \bar{K} \in\{1, . ., K\}, G \in \mathcal{G}}\{ \\
& \left.\sum_{i=1}^{\bar{K}} q_{k(i)}(m T) \bar{\mu}_{i}(F, \bar{K}, G)\right\} .
\end{aligned}
$$

\section{B. Performance of the proposed threshold-based strategy}

The stability region achieved by the threshold-based scheme can be shown to be as follows

Theorem 7. The stability region of the threshold-based scheme when signaling is done every $T$ slots is

$$
\begin{aligned}
& \Lambda_{t h r}(T)= \\
& \left(1-\frac{1}{T}\right) \mathcal{C} \mathcal{H}_{F \in\left\{1, . ., F_{\max }\right\}, \bar{K}=\{1, . ., K\}, G \in \mathcal{G}}\left\{\Lambda^{\prime}(F, \bar{K}, G)\right\},
\end{aligned}
$$

where $\Lambda^{\prime}(F, \bar{K}, G)$ is the convex hull of all points in $\mathbb{R}^{K}$ resulting from all possible permutations of the numbers $\bar{\mu}_{i}(F, \bar{K}, G)$.

The proof goes in the same line as in [12] following the main method of e.g. [14], that is proving first that the stated stability region is the outer bound of what is achievable and then use Lemma 4 along with the fact that (2) maximizes the negative part of the drift of a quadratic function for system $\mathbf{q}(m)$ to prove that the region is indeed achievable. We skip the proof for brevity.

Finally, one can compare the stability region achieved by the threshold based algorithm to the one achieved by the baseline centralized algorithm. By proceeding in a similar way as in [12], we can show that the condition

$$
T>\frac{T_{s}-\beta_{p}-\beta}{\beta_{0}}
$$




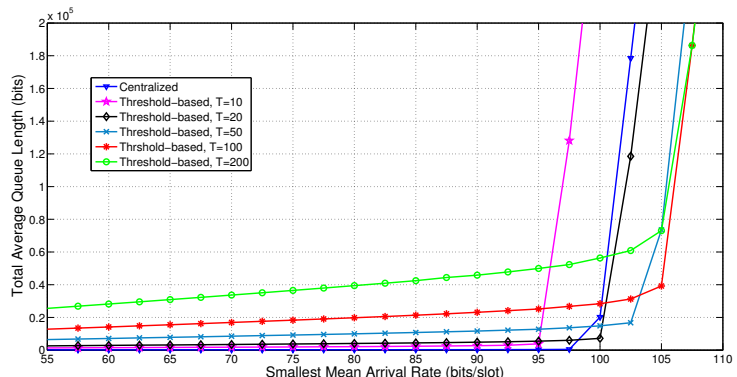

Fig. 1. Average Total Queue Length for Different Mean Arrival Rates with $S=1$ and $P=2$.

where $\beta_{0}=\frac{\log _{2} K}{R_{0}}$ is a sufficient condition for the thresholdbased policy to achieve a bigger stability region than the centralized. We skip here again the proof due to a lack of space. In other words, by simply selecting the broadcast time $T$ such that the above condition is satisfied, our algorithm performs better. Notice that this condition is logical since, according to our algorithm, during the broadcast phase (i.e. at slots $0, T, . . m T,(m+1) T, \ldots)$ no data transmission is performed and these slots are reserved only for signaling. Clearly by reducing the number of slots reserved for broadcast the bit rate will increase. Here, we find explicitly a condition that must satisfy this broadcast time $T$ (in addition to the characterization of the stability region).

\section{Simulation Results}

In order to illustrate the impact of the proposed policy on the stability region of the system, we simulated a single cell system where the BS uses $N=4$ antennas to serve $K=10$ users. Each user has a Poisson traffic with different average arrival rates as $\lambda_{K}:=\lambda$ and $\lambda_{k-1}=1.05 \lambda_{k}$. For simplicity, a threshold rule, where the threshold for the channel gain depends only on the preferred number $F$ of users to be served and threshold $G=\frac{F S}{P}$ is used. The reason for choosing this is that if the channel gain of a user is smaller and $F-1$ other users are served then the rate of this user will be zero. We set the slot duration to be $T_{s}=1000$ channel uses, rate level $R=1$ bit per channel use and overheads for downlink pilot and uplink pilot length per user $\beta_{p}=50$ and $\beta=100$, respectively, while the rate for signalling is set to $R_{0}=0.2$ bits per channel use. We simulated the system for different values of signalling period $T$ and $\lambda$, each simulation lasting $10^{6}$ timeslots. In order to illustrate the stability properties, we present the average total queue lengths, i.e. the quantity $\frac{1}{10^{6}} \sum_{t=0}^{10^{6}-1} \sum_{k=1}^{K} q_{k}(t)$ for $S=P / 2=0.1$ and $S=P=1$ in Figures 1 and 2 respectively.

We can see in the figures that, indeed, by selecting time $T$ higher than the condition in (3), our algorithm performs better than the baseline centralized policy. Notice that increasing $T$ too much will not necessarily keep increasing the stability region. More precisely, by increasing $T$ the stability region will increase but when $T$ takes high values the increase becomes negligible. This can be interpreted using the result in

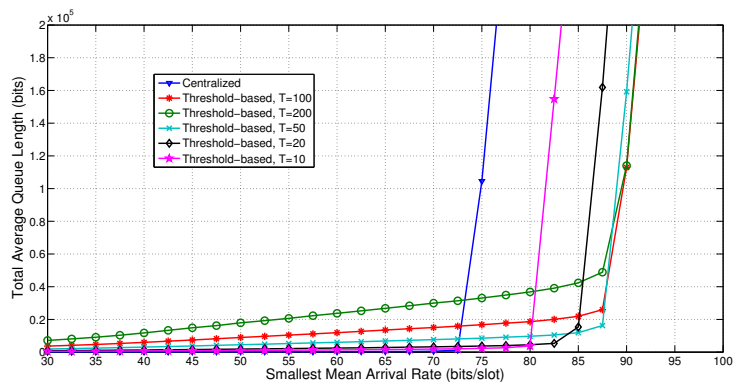

Fig. 2. Average Total Queue Length for Different Mean Arrival Rates with $S=1$ and $P=1$.

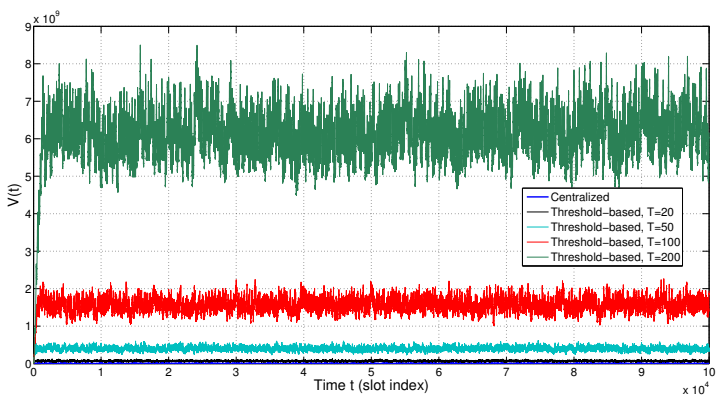

Fig. 3. Evolution the quadratic Lyapunov function for different policies, for $P=S=1$ and $\lambda=70$ bits $/$ slot.

Theorem 7 since the convex hull is multiplied by the $\left(1-\frac{1}{T}\right)$ term. In addition, the threshold-based policy gives higher expansion of the stability region when the SNR threshold for correct decoding is high. This is expected, as the higher the SNR threshold is, the more important accurate channel state information becomes (and the higher probability there is that a user selected under the centralized policy will have a channel that will not allow to support the rate $R$ ).

Another observation from Figures 1 and 2 is that increasing the signalling period $T$ leads to higher average queue lengths for the cases where the system is stable under all policies. This can be explained intuitively by the fact that the centralized policy and policies with low signalling period adapt to real time (or very up to date) queue state information. To examine this in more detail, we show in Figure 3 the evolution of the quadratic Lyapunov function $V(t)=V(\mathbf{q}(t))=\frac{1}{2} \sum_{k=1}^{K} q_{k}^{2}(t)$ over time for $\lambda=70$ bits/slot (i.e. for arrival rates where all queues are stable). Recall that the centralized and threshold based policies try to maximize the negative part of the drift of this Lyapunov function and also that (any) Lyapunov function can be interpreted as a metric of how "far" the queue lengths vector wanders away from the origin (i.e. 0). The Figure shows clearly that, increasing $T$ makes the queues build up in the beginning and the system arrives to a steady state when the queue length vector is further from the origin.

Complimentary to Fig. 3, we plot in Fig. 4 the evolution over time of $q_{1}(t)$ for the same simulation runs. We can see that for the threshold-based schemes the size of this queue 


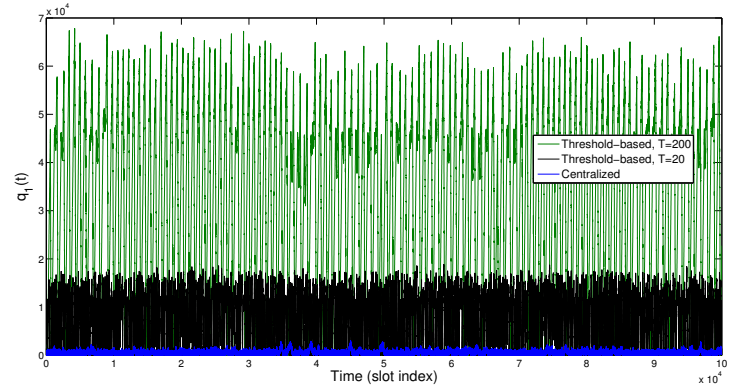

Fig. 4. Evolution of $q_{1}(t)$ under different policies, for $P=S=1$ and $\lambda=70 \mathrm{bits} / \mathrm{slot}$.

tends to build up larger as $T$ grows and then to go down to (almost) zero, this procedure being repeated for the simulation duration. This illustrates the effect of the outdated queue lengths information: Queues that were large in the control slot $m T$ continue to get served in the next $T-1$ slots and queues that were small in $m T$ do not get much service. That means that users with big queues get scheduled most of the time. In the same time, the smaller queues build up, since they do not get much service.

Overall, simulation results imply that increase in the signalling period can increase the stability region of the system at the cost of longer queue lengths and/or more time needed for the Markov chain to reach its steady state.

\section{CONCLUSions}

We developed a scheme for uplink training and user selection in single cell MISO wireless systems where the basic idea is that only users with channel magnitudes above a threshold get scheduled. We show that adjusting the threshold, number of users to get served and set of potential users to be served dynamically, the examined policy can improve the stability region with respect to the centralized policy (where the users are scheduled for training based on the channel statistics). Furthermore, we characterized the stability region of our scheme and the baseline centralized policy.

\section{ACKNOWLEDGEMENTS}

This article has been supported by the ERC Grant 305123 MORE (Advanced Mathematical Tools for Complex Network Engineering). The research of M. Assaad is funded by the european Celtic project SHARING. Part of the work was carried out when the first author was with Alcatel-Lucent Bell Labs France.

\section{APPENDIX}

\section{PROOF OF LEMMA 6}

Proof the first part: Notice that here only one user is to be scheduled (since $F(m)=1$ ). This means that the $i$-th user in the ordering gets scheduled if (i) his channel magnitude is above the threshold and (ii) the channel magnitudes of the $i-1$ users before him are below the threshold. In addition, if this user is scheduled the contention period stops right after, i.e. lasts for $i$ minislots.
Proof of the second part: We now deal with the case where $2 \leq F(m)=F \leq F_{\max }$. The mean rate of the $i$-th user in the ordering can be written as follows:

$\bar{\mu}_{k(i)}(F, \bar{K}, G)=$

$\mathbb{P}\{F$ users above $G, k(i) \in \mathcal{F}\} \mathbb{E}\left\{\bar{\mu}_{k(i)} \mid F\right.$ above $G$,

$k(i) \in \mathcal{F}\}$

$+\sum_{F^{\prime}=1}^{F-1} \mathbb{P}\left\{F^{\prime}\right.$ users above $\left.G, k(i) \in \mathcal{F}\right\} \mathbb{E}\left\{\bar{\mu}_{k(i)} \mid F^{\prime}\right.$ above $G$,

$k(i) \in \mathcal{F}\}$

$:=\hat{\mu}_{i}(F)+\sum_{F^{\prime}=1}^{F-1} \hat{\mu}_{i}\left(F^{\prime}\right)$.

For the rest of the proof, we will denote the event that user $k(i)$ is scheduled as $k(i) \in \mathcal{F}$. For the first term of the above equation, which corresponds to the event that $F(m)=F$ users get scheduled in the end, we have

$$
\begin{aligned}
\hat{\mu}_{i}(F)= & \sum_{m=F}^{\bar{K}} \mathbb{P}\{M=m, k(i) \in \mathcal{F},|\mathcal{F}|=F\}\left[T_{s}-\left(\beta_{p}\right.\right. \\
& +\beta F+m)]^{+} \mathbb{E}\left\{r_{k(i)}(t) \mid F, g_{k(i)}(t)>G\right\}
\end{aligned}
$$

where $M$ denotes the duration of the contention period (i.e. how many minislots are used till $F$ users are found with the channel magnitude above the threshold). Since $k(i)$ should be in the set of users that are scheduled, the contention period should not stop before his minislot, that is

$$
\mathbb{P}\{M=m, k(i) \in \mathcal{F},|\mathcal{F}|=F\}=0, \forall 0 \leq m<i .
$$

In addition, we should also have $m \geq F$. Combined with the above, it implies that $m \geq \max \{F, i\}$. Taking also into account that, by definition $\mathbb{E}\left\{r_{k(i)}(t) \mid F, g_{k(i)}(t)>G\right\}=$ $\bar{r}_{d}(F, G)$, the sum in (5) can be then rewritten as follows:

$$
\begin{aligned}
& \hat{\mu}_{i}(F)= \\
& \mathbb{1}_{\{i \geq F\}}\left[T_{s}-\left(\beta_{p}+\right.\right. \\
& \beta F+i]^{+} \mathbb{P}\{M=i, k(i) \in \mathcal{F},|\mathcal{F}|=F\} \bar{r}_{d}(F, G)+ \\
& \quad \sum_{m=\operatorname{Kax}\{F, i+1\}}^{\bar{K}} \mathbb{P}\{M=m, k(i) \in \mathcal{F},|\mathcal{F}|=F\}\left[T_{s}-\left(\beta_{p}\right.\right. \\
& +\beta F+m)]^{+} \bar{r}_{d}(F, G) .
\end{aligned}
$$

The first term of (6) corresponds to the case where $k(i)$ is the last user to send the signal for which the channel gain is greater than $G$. In this case, exactly $F-1$ out of the $i-1$ previou users must have channel gain above $G$, therefore

$$
\begin{aligned}
\mathbb{P}\{M=i, k(i) \in \mathcal{F},|\mathcal{F}|=F\}= \\
\left(\begin{array}{c}
i-1 \\
F-1
\end{array}\right) p^{F}(G)(1-p(G))^{i-F}
\end{aligned}
$$


For the second term of (6), we note that the event in the probability is equivalent to the union of events where (i) the channel magnitude of user $k(i)$ is above $G$ (ii)the channel magnitude of user $k(m)$ is above $G$ and (iii) $f$ users with lower order than $i$, that is between and including $k(1)$ and $k(i-1)$ and $F-f-2$ between and including $k(i+1)$ and $k(m-1)$ have channel magnitudes above the threshold, for all values of $f$. The values $f$ is allowed to take should satisfy the following properties:

$$
\begin{aligned}
& 0 \leq f \leq i-1 \\
& f \leq F-2 \\
& F-(f+2) \leq m-1-i \Longrightarrow f \geq F-1-(m-i) .
\end{aligned}
$$

The first condition in (8) comes from the fact that the number of users before the $i$-th user is $i-1$, the second from the fact that since $k(i)$ and $k(m)$ should both be scheduled, not more than $F-2$ users higher in the ranking of $k(i)$ should have channel magnitudes above the threshold and the third because there are left $m-i$ users in the ranking between, and not including users $k(i)$ and $k(m)$. We thus have

$$
\begin{aligned}
& \mathbb{P}\{M=m, k(i) \in \mathcal{F},|\mathcal{F}|=F\}= \\
& p^{2}(G) \sum_{f=\max \{0, F-1-m+i\}}^{\min \{F-2, i-1\}} \mathbb{P}\{f \text { in }\{k(1), \ldots, k(i-1) \text { above } G, \\
& F-(f+2) \text { in }\{k(i+1), \ldots, k(m-1) \text { above } G\},
\end{aligned}
$$

which, since the channels are i.i.d. among users reduces to

$$
\begin{aligned}
& \mathbb{P}\{M=m, k(i) \in \mathcal{F},|\mathcal{F}|=F\}= \\
& p^{2}(G) \sum_{f=\max \{0, F-1-m+i\}}^{\min \{F-2, i-1\}} \mathbb{P}\{f \text { out of } i-1 \text { above } G\} \\
& \mathbb{P}\{F-(f+2) \text { out of } m-1-i \text { above } G\} .
\end{aligned}
$$

The event in the first term inside the sum happens in $\left(\begin{array}{c}i-1 \\ f\end{array}\right)$ possible ways with probability $p^{f}(G)(1-p(G))^{i-1-f}$ each, while the event in the second term happens in $\left(\begin{array}{c}m-1-i \\ F-(f+2)\end{array}\right)$ ways, with probability $p^{F-(f+2)}(G)(1-p(G))^{m-i-(F-(f+1))}$ each. Replacing and taking into account that $F \leq m$ (all $F$ users should get scheduled) and we get eventually

$$
\begin{gathered}
\mathbb{P}\{M=m, k(i) \in \mathcal{F},|\mathcal{F}|=F\}=p(G)^{F}(1-p(G))^{m-F} \\
\sum_{f=\max \{0, F-1-m+i\}}^{\min \{F, i\}-1}\left(\begin{array}{c}
i-1 \\
f
\end{array}\right)\left(\begin{array}{c}
m-1-i \\
F-(f+2)
\end{array}\right),
\end{gathered}
$$$$
\text { for } \max \{F, i+1\} \leq m \leq \bar{K}
$$

We now turn to the second term of (4), i.e. the sum $\sum_{F^{\prime}=1}^{F-1} \hat{\mu}_{i}\left(F^{\prime}\right)$. This is in fact due to the probability that less than $F$ users can have channel magnitude above the threshold. The event that exactly $F^{\prime}<F$ users have channel magnitude above the threshold and user $k(i)$ is scheduled is the same as user $k(i)$ having channel magnitude over the threshold and exactly $F^{\prime}-1$ out of the remaining $\bar{K}-1$ users do. This event can happen in $\left(\begin{array}{c}\bar{K}-1 \\ F^{\prime}-1\end{array}\right)$ combinations, each having a probability of $p\left(g^{*}\right) p^{F^{\prime}-1}\left(g^{*}\right)\left(1-p\left(g^{*}\right)\right)^{\bar{K}-F^{\prime}}$. On the other hand, if less than $F$ users are above the threshold then all $\bar{K}$ minislots are used, therefore the contention period lasts for $\bar{K}$ channel uses. Finally, $F^{\prime}$ users participate in the uplink training. We then have

$$
\begin{aligned}
& \hat{\mu}_{i}\left(F^{\prime}\right)=\left(\begin{array}{l}
\bar{K}-1 \\
F^{\prime}-1
\end{array}\right) p^{F^{\prime}}(G)(1-p(G))^{\bar{K}-F^{\prime}} \\
& \quad\left[T_{s}-\left(\beta_{p}+\bar{K}+\beta F^{\prime}\right)\right]^{+} \mathbb{E}\left\{r_{k(i)}(t) \mid F^{\prime}, g_{k(i)}(t)>G\right\} .
\end{aligned}
$$

The result follows combining the above with (4), (5), (6), (7) and (9).

\section{REFERENCES}

[1] D. Gesbert, M. Kountouris, R. Heath, C.-B. Chae, and T. Salzer, "Shifting the MIMO paradigm," IEEE Signal Process. Mag., vol. 24, no. 5, pp. 36-46, 2007.

[2] H. Boche and M. Wiczanowski, "The interplay of link layer and physical layer under MIMO enhancement: benefits and challenges," IEEE Wireless Commun. Mag., vol. 13, no. 4, pp. 48-55, Aug. 2006.

[3] M. Kobayashi and G. Caire, "An iterative water-filling algorithm for maximum weighted sum-rate of Gaussian MIMO-BC," IEEE J. Sel. Areas Commun., vol. 24, no. 8, pp. 1640-1646, Aug. 2006.

[4] D. Love, R. Heath, V. Lau, D. Gesbert, B. Rao, and M. Andrews, "An overview of limited feedback in wireless communication systems," IEEE J. Sel. Areas Commun., vol. 26, no. 8, pp. 1341 -1365, Oct. 2008.

[5] M. Kobayashi, N. Jindal, and G. Caire, "Training and Feedback Optimization for Multiuser MIMO Downlink," IEEE Trans. Commun., vol. 59, no. 8, pp. 2228-2240, Aug.t 2011.

[6] K. Huang and V. Lau, "Stability and delay of zero-forcing SDMA with limited feedback," IEEE Trans. Inf. Theory, vol. 58, no. 10, pp. 64996514, Oct. 2012.

[7] D. Gesbert and M.-S. Alouini, "How much feedback is multi-user diversity really worth?" in IEEE Int. Conf. Commun. (ICC), vol. 1, Paris, France, 2004, pp. 234-238.

[8] V. Hassel, D. Gesbert, M.-S. Alouini, and G. Oien, "A Threshold-Based Channel State Feedback Algorithm for Modern Cellular Systems," IEEE Trans. Wireless Commun., vol. 6, no. 7, pp. 2422 -2426, Jul. 2007.

[9] M. Karaca, Y. Sarikaya, O. Ercetin, T. Alpcan, and H. Boche, "Joint Opportunistic Scheduling and Selective Channel Feedback," IEEE Trans. Wireless. Commun., vol. 12, no. 6, pp. 3024-3034, Jun. 2013.

[10] T. Tang, R. W. Heath, S. Cho, and S. Yun, "Opportunistic feedback for multiuser MIMO systems with linear receivers," IEEE Trans. Commun., vol. 55, no. 5, pp. 1020-1032, 2007.

[11] A. Destounis, M. Assaad, M. Debbah, and B. Sayadi, “ Traffic-Aware Training and Scheduling for the 2-user MISO Broadcast Channel," in IEEE Int. Symp. Inf. Theory (ISIT), Honolulu, HI, USA, 2014.

[12] —, "Traffic-Aware Feedback and Scheduling for Wireless MISO Downlink Systems," IEEE Trans. Inf. Theory, 2014, under revision.

[13] A. Gopalan, C. Caramanis, and S. Shakkottai, "On wireless scheduling with partial channel-state information," IEEE Transactions on Information Theory, vol. 58, no. 1, pp. 403 -420, Jan. 2012.

[14] L. Tassiulas and A. Ephremides, "Stability properties of constrained queueing systems and scheduling policies for maximum throughput in multihop radio networks," IEEE Trans. Autom. Control, vol. 37, no. 12, pp. $1936-1948$, Dec. 1992. 